\title{
miR-493-5p attenuates the invasiveness and tumorigenicity in human breast cancer by targeting FUT4
}

\author{
LIFEN ZHAO $^{1 *}$, XIAOBIN FENG ${ }^{1 *}$, XIAOBO SONG ${ }^{2}$, HUIMIN ZHOU $^{3}$, \\ YONGFU ZHAO ${ }^{4}$, LEI CHENG ${ }^{5}$ and LI JIA $^{1}$ \\ ${ }^{1}$ College of Laboratory Medicine, Dalian Medical University, Dalian, Liaoning 116044, P.R. China; \\ ${ }^{2}$ Department of Medical Biology, Faculty of Health Sciences, University of Troms $\varnothing$, Troms $\varnothing$, Norway; \\ ${ }^{3}$ Department of Microbiology, Dalian Medical University, Dalian, Liaoning 116044; ${ }^{4}$ Department of General Surgery, \\ The Second Affiliated Hospital of Dalian Medical University, Dalian, Liaoning 116027; \\ ${ }^{5}$ Department of Laparoscopic Surgery, The First Affiliated Hospital of Dalian Medical University, \\ Dalian, Liaoning 116011, P.R. China
}

Received February 5, 2016; Accepted March 15, 2016

DOI: 10.3892/or.2016.4882

\begin{abstract}
Breast cancer is a leading cause of cancer-related mortality among women. Altered fucosylation was found to be closely associated with tumorigenesis and metastasis of breast cancer. MicroRNAs (miRNAs) are important regulators of cell proliferation and metastasis, and aberrant miRNA expression has been observed in breast cancer. The present study aimed to evaluate the level of fucosyltransferase IV (FUT4) and miR-493-5p in breast cancer and investigate their relationship. In the present study, we demonstrated the differential expressional profiles of FUT4 and miR-493-5p in 29 clinical breast cancer tissues, matched adjacent tissue samples and two breast carcinoma cell lines (MCF-7 and MDA-MB-231). Briefly, altered expression levels of FUT4 modified the invasive activities and tumorigenicity of the MCF-7 and MDA-MB-231 cells. Further study demonstrated that miR-493-5p plays a role as a suppressor in breast cancer cell invasion and tumorigenicity. Moreover, the expression levels of miR-493-5p were inversely proportional to those of FUT4 both at the mRNA and protein levels. Luciferase reporter assays confirmed that miR-493-5p bound to the 3'-untranslated (3'-UTR) region of FUT4, and inhibited the expression of FUT4 in breast cancer cells. Taken together, our data suggest that FUT4 may have a potential role in the treatment of breast cancer, as well as miR-493-5p is a novel regulator of invasiveness and tumorigenicity of breast
\end{abstract}

Correspondence to: Dr Li Jia, College of Laboratory Medicine, Dalian Medical University, 9 Lvshunnan Road, Xiduan, Dalian, Liaoning 116044, P.R. China

E-mail: jiali0386@sina.com

*Contributed equally

Key words: FUT4, breast cancer, miR-493-5p, breast cancer cell lines, invasion, tumorigenicity cancer cells through targeting FUT4. The miR-493-5p/FUT4 pathway has therapeutic potential in breast cancer.

\section{Introduction}

Breast cancer is the most common invasive cancer and the second cause of cancer-related death in women $(1,2)$. Each year more than a half a million new cases of breast cancer are diagnosed in the US and Europe (3), and in China. Breast cancer is a very heterogeneous disease. Some patients are cured by the surgical removal of the primary tumor while other patients suffer from metastasis and progression of the disease, despite adjuvant therapy. Therefore, it is essential to develop effective and safer therapeutic modalities against breast cancer.

Glycosylation is one of the important steps of protein post-translational modifications, and $\sim 50 \%$ of proteins are glycosylated (4). Protein glycosylation plays a role in a variety of cellular biological functions, such as cell-cell and cell-substrate adhesion, membrane organization, cell immunogenicity and protein targeting (5). Specific changes in the glycosylation patterns of cell surface glycoprotein have been shown to enhance the metastatic potential of tumor cells. Aberrant expression of fucosylated glycans has also been detected in various types of tumors.

The fucosyltransferase (FUT) family is a group of fucosylation synthases that catalyze the transfer of L-fucose (Fuc) from an activated GDP- $\beta$-L-Fuc to various acceptor molecules such as $N$-acetyllactosamine. The transfer of Fuc residue from the donor substrate, GDP-Fuc, is catalyzed to the oligosaccharide acceptor in a1,2-(FUT1 and FUT2), a1,3/4-(FUT3, FUT4, FUT5, FUT6, FUT7, FUT9, FUT10 and FUT11) and a1,6-linkage (FUT8). Frequent fucosylation, the final step within the glycosylation machinery, results in glycans that are involved in various cellular processes such as cell-cell recognition, adhesion and inflammation or tumor metastasis. Fucosylation is suggested to have paramount importance in the invasion and metastatic process of cancer stem cells (CSCs) (6), for example increasing FUT4 and FUT7 expression promoted 
neoplastic cell proliferation and hepatocellular carcinoma cell growth in vitro, respectively, and reducing FUT3/6 expression suppressed colon carcinoma cell proliferation $(7,8)$.

MicroRNAs (miRNAs) comprise a class of small noncoding RNAs implicated in post-transcriptional RNA regulation. These RNA molecules are $22 \mathrm{nt}$ in length (9). By binding to complementary sequences in the 3'UTRs of targeted mRNAs, miRNAs degrade or inhibit their translation and regulate a range of cellular functions such as differentiation, proliferation, apoptosis and migration of tumor cells (10). Accumulating evidence demonstrates that various types of miRNAs participate in the regulation of tumorigenesis and metastasis $(11,12)$. Recent studies support the assumption that modulating the levels of miR-146a or miR-146b could have therapeutic potential to suppress breast cancer metastasis (13). It was reported for the first time that anti-miR-17 molecules reduced breast cancer cell migration in vitro and metastasis in vivo (14). miR-184 was identified as a putative breast tumor suppressor in pubertal mouse mammary gland (15).

In the present study, we evaluated the expression level of the FUT4 gene in the MCF-7 and MDA-MB-231 cell lines and clinical breast cancer samples. We also investigated whether FUT4 participates in the regulation of tumor invasion and tumorigenicity. In addition, the present study aimed to determine the association between miR-493-5p and FUT4 in human breast cancer, in order to provide a better understanding of the mechanisms underlying breast cancer invasion and tumorigenicity.

\section{Materials and methods}

Cell culture. Cell lines MDA-MB-231 and MCF-7 were obtained from KeyGen Co. (Nanjing, China) and cultured in Dulbecco's modified Eagle's medium (DMEM), $10 \%$ fetal bovine serum (FBS) and $1 \%$ penicillin-streptomycin (both from Gibco, Grand Island, NY, USA) at $37^{\circ} \mathrm{C}$ in a humidified atmosphere under $5 \% \mathrm{CO}_{2}$.

Patient tissue collection and RNA extraction. Twenty-nine breast cancer and matched adjacent tissue samples were resected from patients at the Second Affiliated Hospital of Dalian Medical University (Dalian, China) from July 2011 to June 2014. Informed consent forms and the entire protocol were approved by the Ethics Committee of the Second Affiliated Hospital of Dalian Medical University. The samples were stored at $-80^{\circ} \mathrm{C}$, and total RNA was extracted with TRIzol reagent (Invitrogen, Carlsbad, CA, USA).

Quantitative real-time PCR analysis. The concentration and quality of each total RNA sample was determined using A260/ A280 spectrophotometric reading. cDNA was synthesized with TaqMan reverse transcription reagents (Applied Biosystems, Branchbury, NJ, USA), following the manufacturer's recommendations. Real-time PCR was carried out using 7500 Fast Real-time PCR system (Applied Biosystems). Reactions were run in 3 independent experiments. The relative expression level of FUT4 was normalized to GAPDH. The primer sequences were: 5'-TCCTACGGAGAGGCTCAG-3' and 5'-TCCTCG TAGTCCAACACG-3'. RT-PCR for miR-493-5p was performed using Real-Time PCR Universal
Reagent (GenePharma, Shanghai, China). U6 was used as an internal control.

Western blot analysis. Protein was extracted from the cells using 1X radioimmunoprecipitation assay lysis buffer (Santa Cruz Biotechnology, Santa Cruz, CA, USA), subjected to SDS-PAGE, and then transferred to polyvinylidene difluoride membranes. The membranes were blocked in 5\% skimmed milk for $2 \mathrm{~h}$, probed with the antibody against human FUT4 (1:1,000 dilution; Abcam, Cambridge, UK) or GAPDH $(1: 1,000$ dilution) at $4^{\circ} \mathrm{C}$ overnight, and with peroxidase-conjugated secondary antibody (1:1,000 dilution) (both from Santa Cruz Biotechnology), and then visualized by chemiluminescence (GE Healthcare, Fairfield, CT, USA).

In vivo tumorigenesis. All animal experiments were performed according to the protocol of the Dalian Committee on Animal Care using 5- to 6-week-old male athymic nude mice. Cells $\left(1 \times 10^{7}\right)$ were subcutaneously injected into the right flank of each nude mouse. The length (L) and width (W) of each tumor were measured every 7 days with calipers, and the volume was calculated.

Lentivirus production and infection. The FUT4 coding sequence (CDS) was obtained and inserted into the NotI and BamHI sites of the pGLV5/H1/GFP+Puro lentiviral plasmid, respectively. The FUT4 shRNA sequences were inserted into the BamHI and EcoRI sites of the pGLV3/H1/GFP+Puro lentiviral plasmid. Lentiviral plasmids were co-transfected with PG-P1-VSVG, PG-P2-REV and PG-P3-RRE plasmids into 293T cells (Invitrogen), and virus-containing supernatants were prepared according to the manufacturer's instructions. For the lentiviral infection, the cells cultured in 6-well tissue culture plates were infected with the lentiviral vectors at a multiplicity of infection of 40 for $24 \mathrm{~h}$. The medium was replaced with fresh complete medium. After 2 days, the cells were observed by fluorescence microscopy to confirm that $>90 \%$ of the cells were GFP-positive. Subsequently, the GFP-positive cells were screened by addition of $5 \mu \mathrm{g} / \mathrm{ml}$ puromycin.

In vitro ECM invasion assay. Cell invasion was assessed using the Matrigel invasion chamber (Corning, Corning, NY, USA) in triplicate. The cells $\left(1.0 \times 10^{5}\right)$ were harvested in serum-free medium containing $0.1 \%$ BSA and plated to the upper chamber precoated with Matrigel. Medium containing $10 \%$ FBS in the lower chamber served as the chemoattractant. After the cells were incubated at $37^{\circ} \mathrm{C}$ in a humidified incubator with $5 \% \mathrm{CO}_{2}$ for $24 \mathrm{~h}$, the non-invading cells were removed with cotton swabs. The invasive cells that had attached to the lower surface of the inserted membrane were fixed in $100 \%$ methanol at room temperature and stained with Wright-Giemsa. The number of invasive cells on the lower surface of the membrane was then counted under a microscope.

Luciferase reporter assay. A pmirGLO Dual-Luciferase miRNA target expression vector was used for 3'UTR luciferase assays (Promega, Madison, WI, USA). The target genes of miRNA-493-5p were selected based on target scan algorithms (microRNA.org (http://www.microrna. 
A

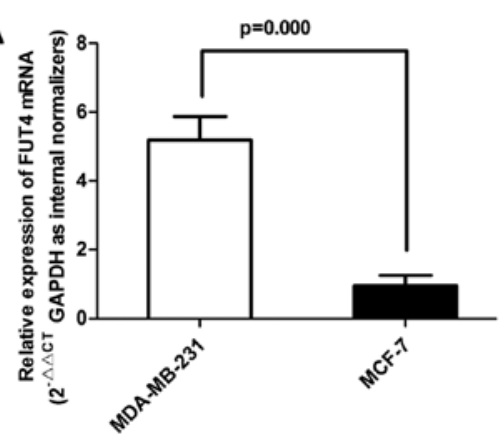

C

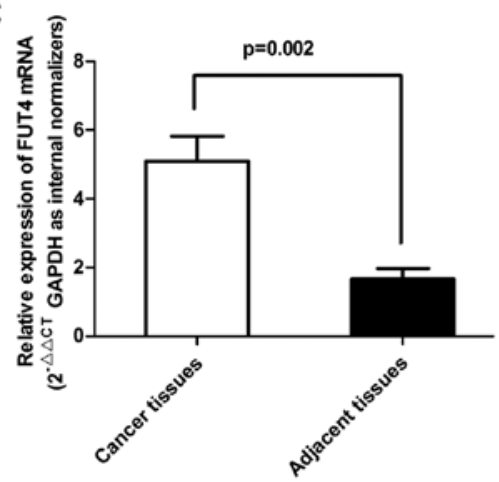

B

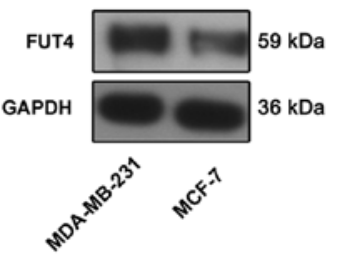

D

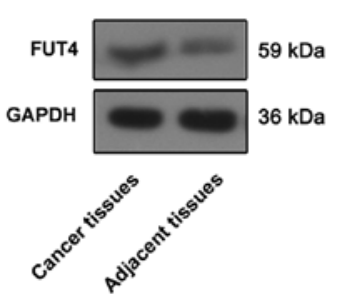

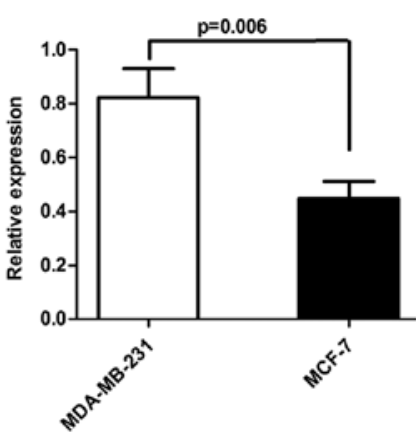

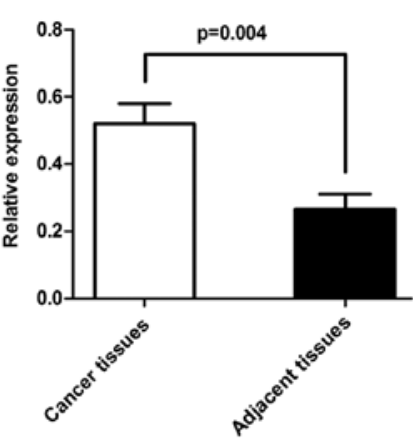

Figure 1. Differential expression of the FUT4 gene in human breast cancer cell lines and tissues. (A and B) The levels of FUT4 were analyzed in breast cancer cell lines (MCF-7 and MDA-MB-231) via real-time PCR and western blotting. Increase in FUT4 was observed in the MDA-MB-231 cells. (C and D) The levels of FUT4 were analyzed in tissues. The primary breast cancer tissues expressed higher expression of FUT4 both at the mRNA and protein levels than levels in the matched adjacent tissue samples $\left({ }^{*} \mathrm{P}<0.05\right)$. Data are the mean $\pm \mathrm{SD}$ of triplicate determinants.

org/microrna/home.do), MicroCosm (http://www.ebi. ac.uk/enright-srv/microcosm/htdocs/targets/) and TargetScan (http://www. targetscan. org/). For 3'UTR luciferase assay, the cells were co-transfected with hsa-miR-493-5p mimics or pmirGLO Dual-Luciferase miRNA target expression vectors and the wild-type or mutant target sequence using Lipofectamine 2000. Luciferase assay was performed using the Dual-Luciferase ${ }^{\circledR}$ reporter assay system (Promega) after transfection at $48 \mathrm{~h}$. Data are presented as the mean value $\pm \mathrm{SD}$ for triplicate experiments.

Statistical analysis. Statistical analyses were carried out using the SPSS 17.0 program. An independent sample t-test was run to analyze the significance of the differences found. P-value $<0.05$ was considered to indicate a statistically significant result. All data are presented as the mean $\pm \mathrm{SD}$.

\section{Results}

Expression of FUT4 in human breast cancer cell lines and tissues. We first determined the expression of FUT4 in breast cancer cell lines, primary breast cancer and the matched adjacent tissue samples. As shown in Fig. 1A and B, the FUT4 (5.76-fold) expression level was significantly higher in the MDA-MB-231 cell line than that in the MCF-7 cell line. Furthermore, analysis of FUT4 expression in pairs of the primary breast cancer and matched adjacent tissue samples revealed that FUT4 was upregulated in the primary breast cancer tissue samples (Fig. 1C and D). These data support the assumption that the differential expression of FUT4 may be associated with breast cancer.
Effect of FUT4 overexpression or silencing on the invasive ability and tumorigenicity of breast cancer cells. To test whether FUT4 plays a role in regulating invasion, we infected MCF-7 cells with lentiviral vectors containing FUT4. After infection, the MCF-7/FUT4 cells expressed high exogenous FUT4 (Fig. 2A and B) in comparison with the uninfected controls or GFP-expressing cells.

Overexpression of FUT4 increased the invasive ability of the MCF-7 cells in vitro (Fig. 2C). In order to further confirm that FUT4 affects invasive ability, we used shRNA delivered in a lentiviral vector tagged with GFP to regulate FUT4 expression in the MDA-MB-231 cells. Three different shRNA lentiviruses significantly reduced the FUT4 expression $>60 \%$ at the mRNA level and $>50 \%$ at the protein level (Fig. 3A and B). The invasive ability was significantly decreased to $55 \%$ in the MDA-MB-231/FUT4-shRNA cells compared with the invasive ability noted in the control cells (Fig. 3C).

In order to identify whether FUT4 further affects the tumorigenicity in vivo, MCF-7/control, MCF-7/FUT4, MDA-MB-231/control shRNA and MDA-MB-231/ FUT4-shRNA1 were subcutaneously injected into nude mice and tumor formation was monitored. On day 28 , the mice were sacrificed under anesthesia and tumor weights were measured. Tumors grew faster in the MCF-7/FUT4 and MDA-MB-231/ control shRNA groups when compared with the rates in the MCF-7/control and MDA-MB-231/FUT4-shRNA1 groups, respectively (Figs. 2D and 3D). These data suggest that FUT4 enhanced the cell growth in vivo.

Differential expression of miR-493-5p in breast cancer cell lines and tissues. To understand the mechanisms by which 
A

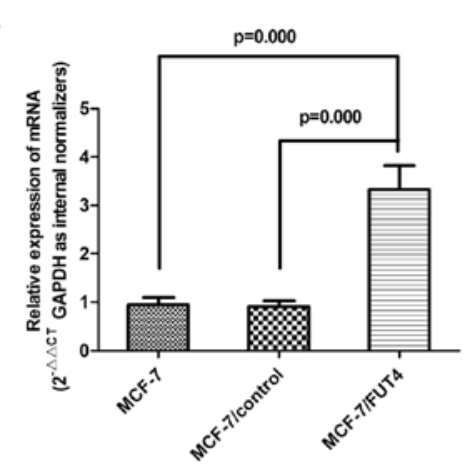

C

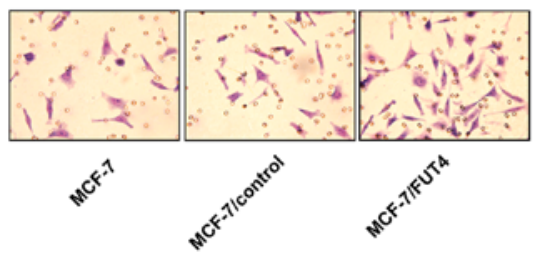

B
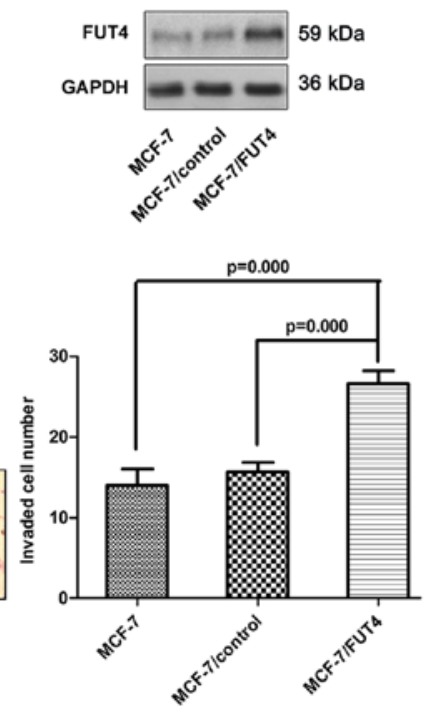

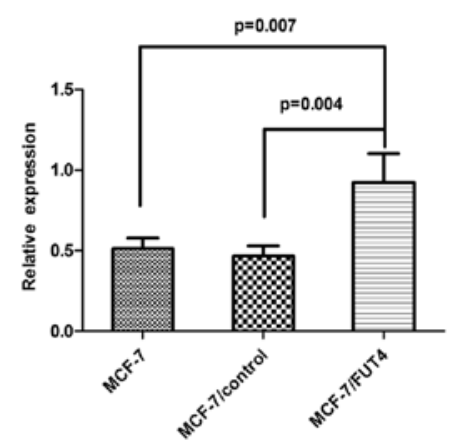

D

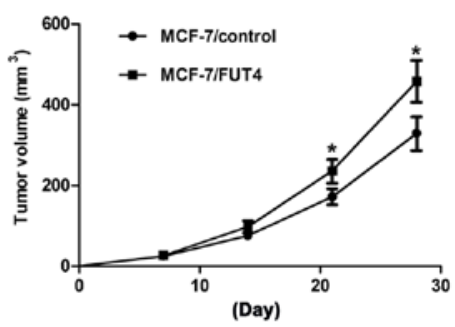

Figure 2. Effect of overexpression of FUT4 on the invasive ability and tumorigenicity of breast cancer cells in vitro and in vivo. (A) Overexpression of FUT4 in cells was analyzed following transfection of lentiviral vectors containing FUT4. FUT4 was detected by RT-PCR after lentiviral vector transfection. (B) Expression of the FUT4 protein was detected by western blotting. (C) The average number of cells that invaded through the filter was counted by ECMatrix gel analysis. (D) The growth curves of tumors after injection of MCF-7/control and MCF-7/FUT4 cells in nude mice ("P<0.05). Tumor sizes were measured every 7 days during a 28 -day period. Data are the mean \pm SD of triplicate determinants.

A

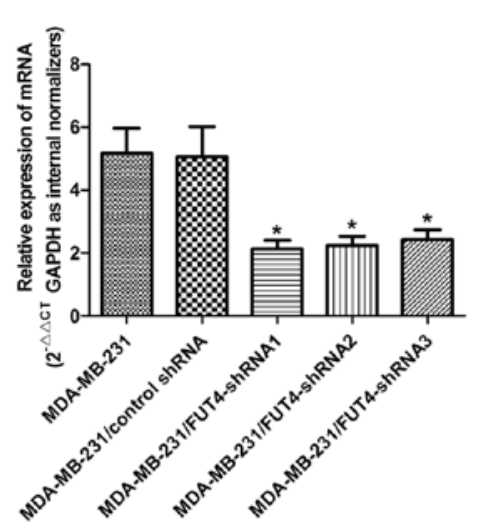

C

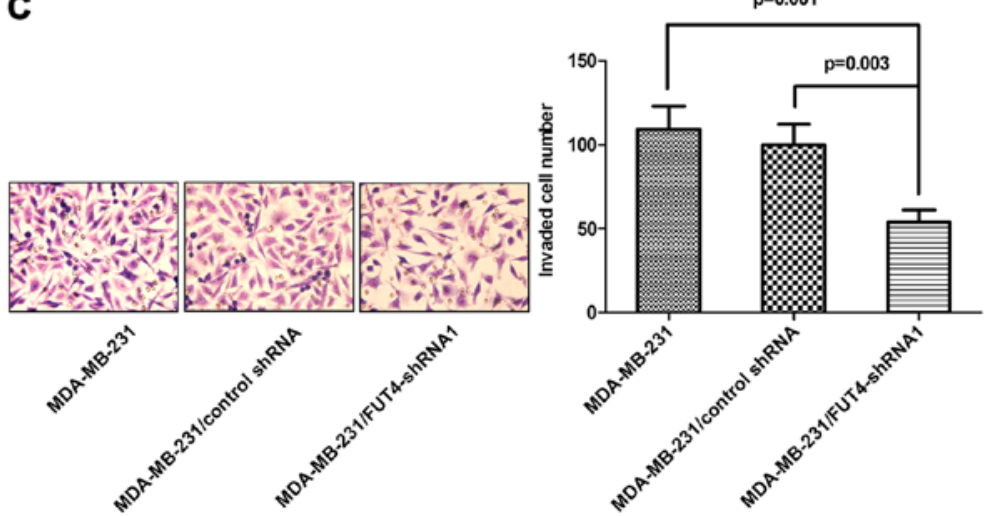

B

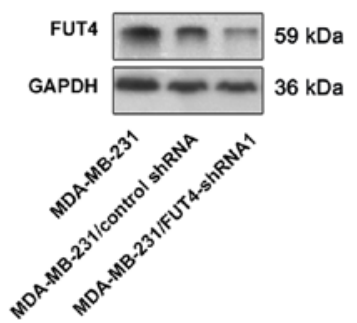

D

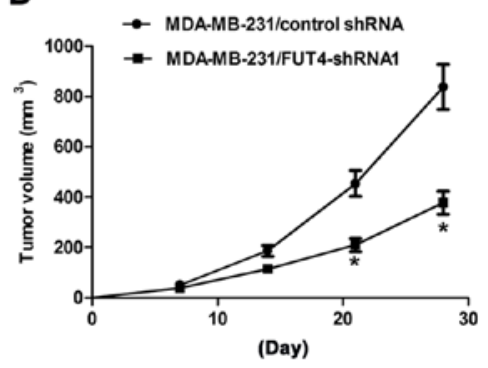

Figure 3. Effects of the silencing of FUT4 on the invasiveness and tumorigenicity of breast cancer cells in vitro and in vivo. (A) The mRNA level of FUT4 was investigated by RT-PCR after shRNA transfection. (B) Expression of the FUT4 protein was detected by western blotting. (C) In vitro ECMatrix gel analysis was performed. (D) Tumor volumes were measured every 7 days during the 28 -day period ( $\mathrm{P}<0.05)$. Data are the mean \pm SD of triplicate determinants. 

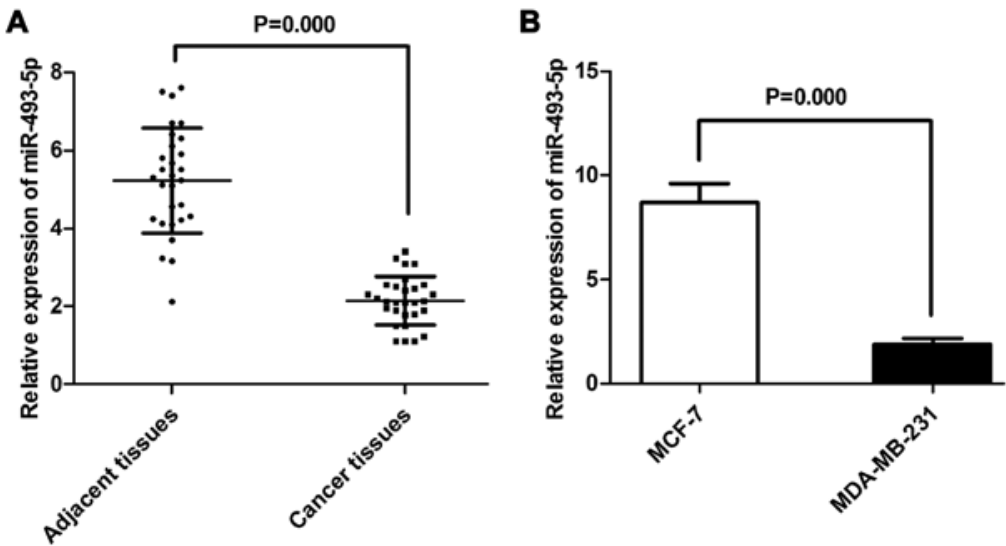

Figure 4. Differential expression of miR-493-5p in breast cancer cell lines and tissues. (A) A comparison of miR-493-5p expression between the primary breast cancer and the matched adjacent tissue samples. (B) The expression of miR-493-5p was examined by real-time PCR in MDA-MB-231 and MCF-7 cells. U6 was included as a control. The miR-493-5p expression was higher in MCF-7 cells than that in the MDA-MB-231 cells, while miR-493-5p was decreased in primary breast cancer tissues in comparison to the matched adjacent tissues ( $\mathrm{P}<0.05)$. Data are the mean $\pm \mathrm{SD}$ of triplicate determinants.

miRNAs execute their function by FUT4, we adopted 3 bioinformatic algorithms (TargetScan, MicroCosm and miRanda) to identify a large number of potential miRNAs. Among these candidates, miR-493-5p was selected for further study. Total RNA was isolated from the cell lines, the breast cancer tissue and the matched adjacent tissue samples, and the miR-493-5p levels were determined using TaqMan real-time PCR. The miR-493-5p expression level was higher in the MCF-7 cells than that in the MDA-MB-231 cells, while the tissue samples showed the same tendency (Fig. 4A and B).

miR-493-5p directly targets and inhibits FUT4 expression. To test whether FUT4 is a direct target of miR-493-5p, luciferase activity assays were performed using luciferase reporters. The 3'-untranslated region (3'-UTR) of the FUT4-mRNA, which contains a predicted target site for miR-493-5p, was cloned into the GP-miRGLO plasmid. In addition, 3 nucleotides within the predicted miR-493-5p target site were mutated in the GP-miRGLO-FUT4-3'-UTR mutant plasmid. When the 3'-UTR of the FUT4 mRNA was involved in the luciferase transcript, forced miR-493-5p expression decreased luciferase activity by $35 \%$ (Fig. 5A), whereas, miR-493-5p mimics had no effect on the luciferase activity of the reporters containing a mutant FUT4 3'-UTR. The results indicated that miR-493-5p targeted the 3'-UTR of the FUT4 mRNA and repressed its expression.

To determine whether FUT4 expression is indeed regulated by miR-493-5p in vitro, the MDA-MB-231 cells were transfected with miR-493-5p agomir, while the MCF-7 cells were transfected with the miR-493-5p antagomir. As expected, miR-493-5p agomir-treated cells showed higher expression of miR-493-5p, and miR-493-5p antagomir inhibited miR-493-5p expression (Fig. 5B and E). The MDA-MB-231 cell line with miR-493-5p expression showed a significant attenuation of FUT4 expression at both the mRNA and protein levels in vitro. Moreover, inhibition of miR-493-5p in MCF-7 cells led to the opposite changes in FUT4 expression (Fig. 5C, D, F and G).

miR-493-5p attenuates the invasiveness in vitro and the tumorigenicity in human breast cancer by targeting FUT4 in vivo. Since the expression levels of miR-493-5p were lower in the MDA-MB-231 cells and breast cancer tissues when compared with the levels in MCF-7 cells and matched adjacent tissues, we examined how alterations in miR-493-5p influence metastatic behavior. MCF-7 and MDA-MB-231 cells were transfected with antagomir or agomir of miR-493-5p, respectively. The effects of the antagomir and the agomir on the invasive rate of MDA-MB-231 and MCF-7 cells were studied with Transwell assay (Fig. 6A and B). Forced miRNA-493-5p overexpression resulted in $41 \%$ reduction in the invasive potential of the MDA-MB-231 cells compared to the control cells. The invasive potential was increased in the MCF-7 cells transfected with the antagomir. The enhanced expression of FUT4 significantly increased or reversed the invasive capabilities of the MCF-7 cells (Fig. 6F and G).

To ascertain whether miR-493-5p could further affect the tumorigenicity in vivo, MDA-MB-231, MDA-MB-231/ $\mathrm{NC}$ and MDA-MB-231/agomir cells were injected into nude mice and tumor formation was monitored. The tumor volume in the MDA-MB-231/agomir group was decreased compared to that noted in the MDA-MB-231 and MDA-MB-231/NC groups (Fig. 6C). Downregulation of FUT4 was also observed in the MDA-MB-231/agomir group (Fig. 6D and E).

\section{Discussion}

Changes in fucosylation can be observed in nearly all malignancies, such as breast (16), HCC (17), colon (18) and lung cancer (19). High incidence of sLex in ER-negative breast tumors coincided with high expression of glycosyltransferases FUT3, FUT4 and ST3GAL6 (20). Previous studies have demonstrated a significant high expression of FUT4 in breast cancer tissues and sera in comparison to normal tissues and sera (21). The present study revealed that the expression profile of the FUT4 gene varied between MDA-MB-231 and MCF-7 cell lines with different metastatic potential. Compared to the MCF-7 cells, MDA-MB-231 cells showed upregulated expression of FUT4 (5.76-fold). Comparison of FUT4 expression levels in 29 pairs of clinical primary breast cancer tissues and their matched adjacent tissues revealed that FUT4 was upregulated in the primary breast cancer tissues. FUT4 with different 
A
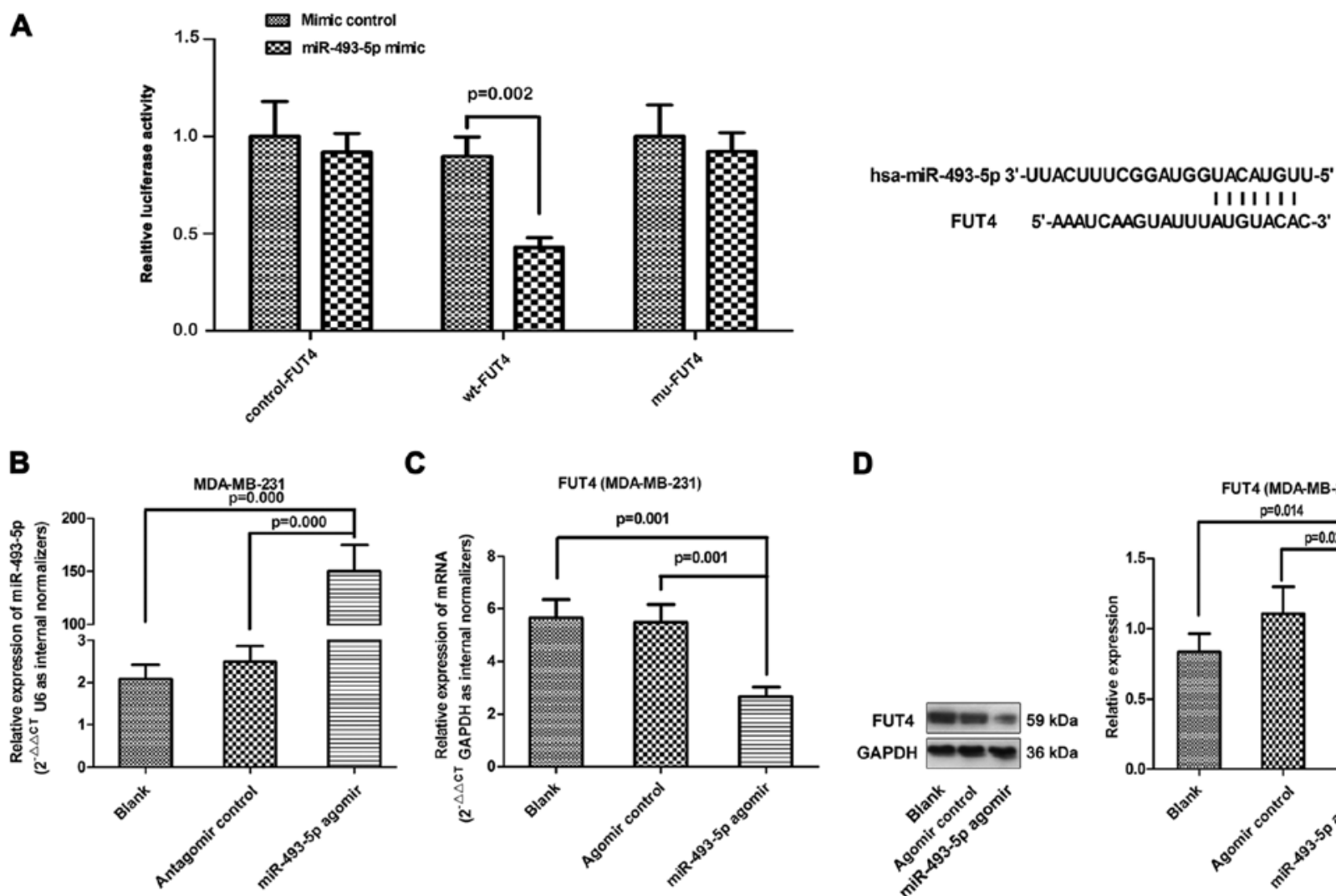

C



D
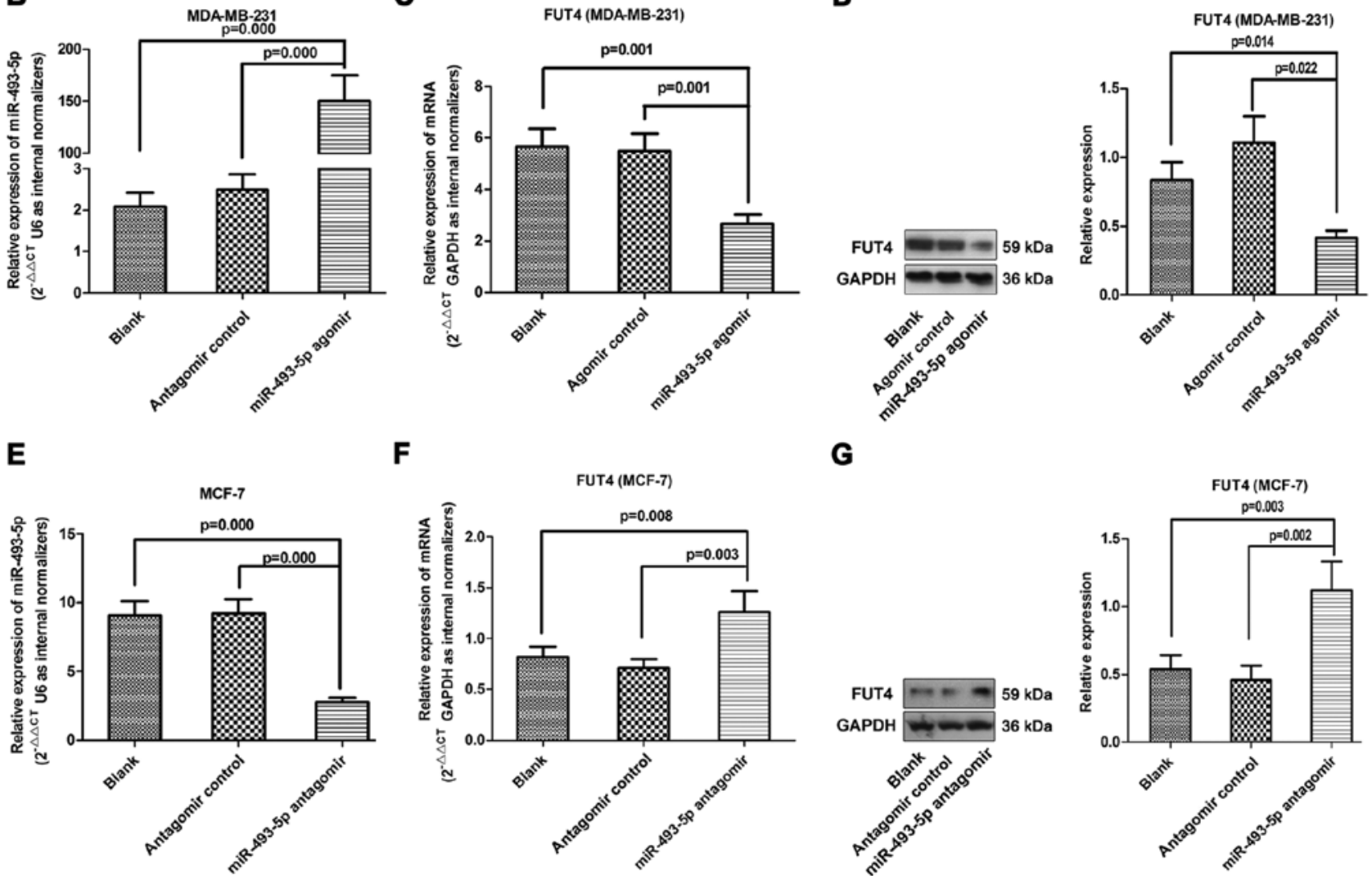

Figure 5. FUT4 is a target gene of miR-493-5p. (A) Dual-luciferase reporter assays were performed by pmir-reporter constructs containing the FUT4 3'-UTR region with the seed sequences or a mutated sequence that was inserted downstream of the luciferase coding sequence. Transfection with a miR-493-5p mimic decreased luciferase activity of the reporter vector containing the 3'-UTR of a wild-type FUT4 ("P<0.05). (B and E) Expression of miR-493-5p was examined after transfection of MDA-MB-231 and MCF-7 cells with miR-493-5p agomir, miR-493-5p agomir control, miR-493-5p antagomir or miR-493-5p antagomir control. (C and F) The mRNA of FUT4 was detected by RT-PCR in cells treated with the agomir or antagomir ( $\left.{ }^{*} \mathrm{P}<0.05\right)$. (D and G) The protein levels of the FUT4 protein were detected by western blotting $\left({ }^{*} \mathrm{P}<0.05\right)$. Data are the mean $\pm \mathrm{SD}$ of triplicate determinants.

expression patterns in the two cell lines and breast cancer tissues imply its role as an indicator and functional regulator of breast cancer metastasis.

A previous study found that stable transfection of antisense sequences directed at the human Lewis $\alpha(1,3 / 1,4)$ FUT gene, FUT3 led to the inhibition of E-selectin-mediated adenocarcinoma cell adhesion (22). Elevated expression of FUT3/6 conferred increased sLex production. sLex ${ }^{+}$cells (with high FUT expression levels) were significantly more invasive than sLex cells (with low FUTs). This suggests an association between FUT expression and invasive activity. Inactivation of the expression of FUT1 and FUT2 reduced cell adhesion efficiency and inhibited cell growth (23). FUT6, a member of the a1,3/4 FUT subfamily, was found to regulate the P13K/ AKT signaling pathway in HCC cells (24). Whether the FUT4 and its encoded protein affect tumor invasion, proliferation and tumorigenicity in breast cancer remains a question. The present study aimed to explore FUT4. The altered level of FUT4 led to mediated invasiveness and tumorigenicity in MCF-7 and MDA-MB-231 cell lines both in vitro and in vivo. Taken together, these data suggest that fucosylation is closely related to the activities of invasion and proliferation in breast cancer cells.

MicroRNAs (miRNAs) are attractive candidates as upstream regulators of metastatic progression since miRNAs can post-transcriptionally regulate entire sets of genes $(25,26)$. 
A

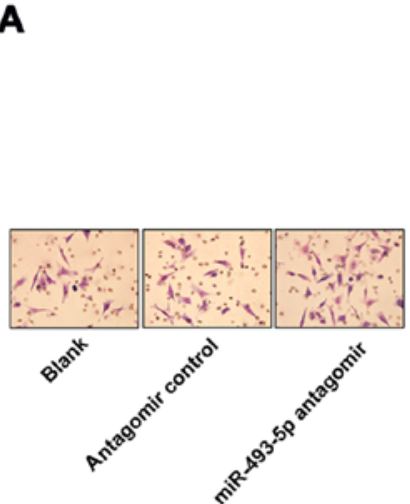

C

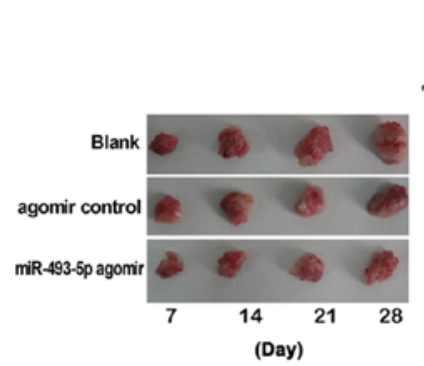

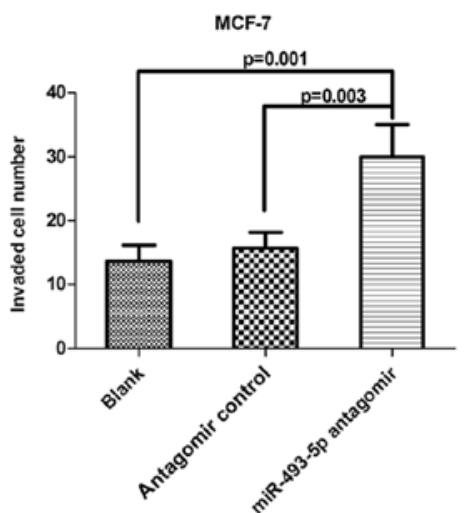

B
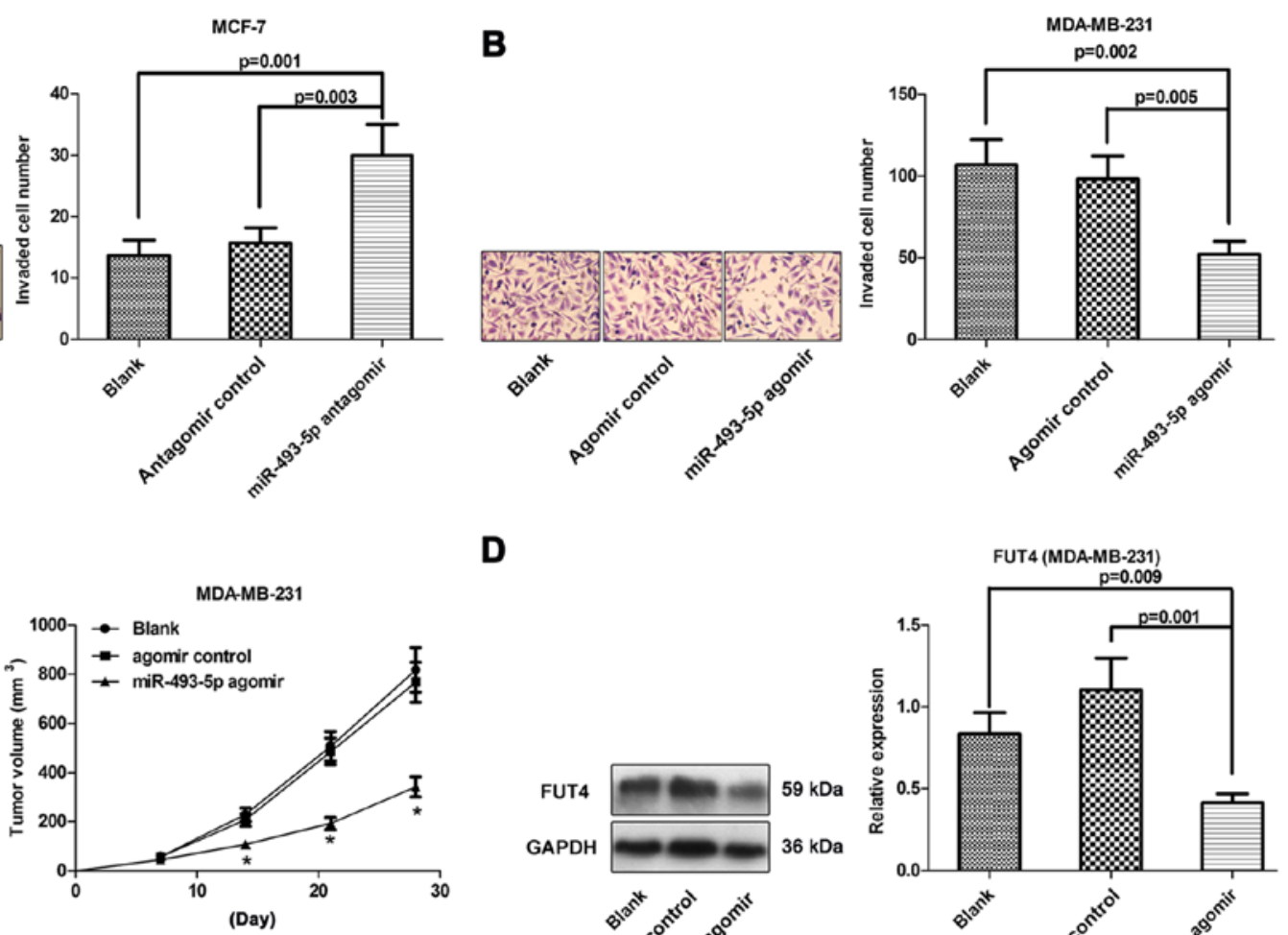

FUT4

D

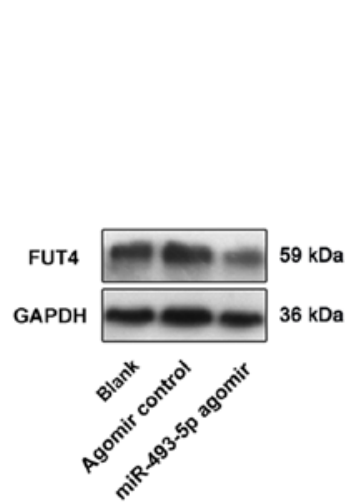



E

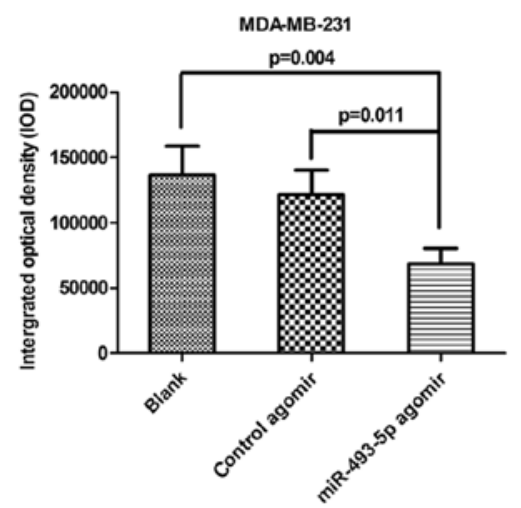

$\mathbf{F}$
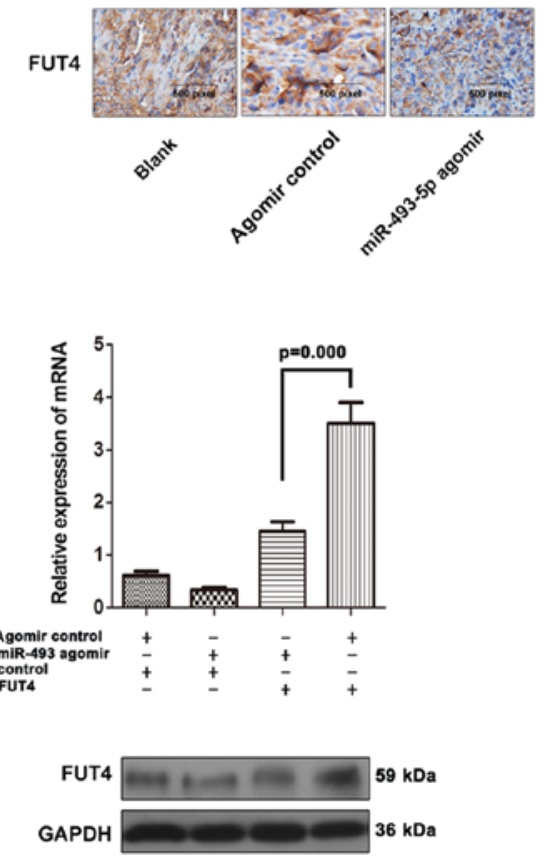

G

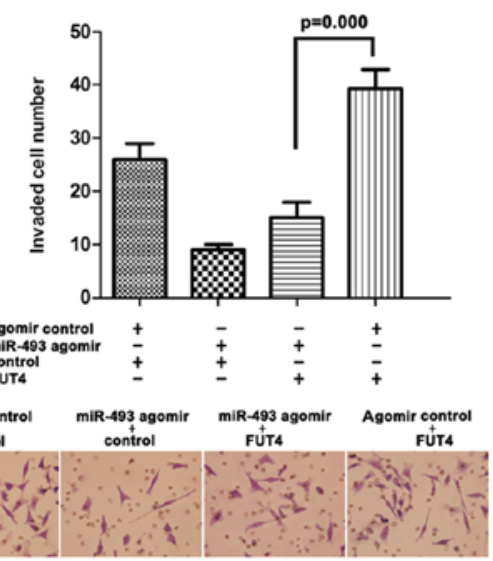

Figure 6. miR-493-5p attenuates the invasiveness and tumorigenicity in human breast cancer by targeting FUT4 both in vitro and in vivo. (A) Knockdown of miR-493-5p increased the invasive ability of the MCF-7 cells ("P<0.05). (B) Overexpression of miR-493-5p decreased the invasive ability of MDA-MB-231 cells ( $\mathrm{P}<0.05)$. (C) Tumor volume was assessed every 7 days. (D and E) The expression levels of FUT4 in nude mice were analyzed by western blotting and immunohistochemistry. Reduced immunohistochemistry staining against FUT4 was found in the MDA-MB-231/agomir group, compared to the MDA-MB231/NC group. (F) MCF-7/control and MCF-7/FUT4 cells were respectively transfected with the miR-493-5p agomir or agomir control. Western blotting showed that MCF-7/FUT4 cells transfected with the miR-493-5p agomir blocked miR-493-5p-induced suppression of FUT4 protein expression. (G) Invasion assays indicated that FUT4 restoration abolished miR-493-5p-induced suppression of invasion and metastasis in MCF-7/FUT4 cells after transfection with the miR-493-5p agomir. The results are expressed as mean \pm SD of 3 independent experiments $(" \mathrm{P}<0.05)$. 
Recent research has revealed the vital roles of miRNAs in tumorigenesis $(27,28)$. miRNAs often act as oncogenes or tumor suppressors, regulating many cellular processes. miR-335 was found to suppress metastasis and migration through targeting of the progenitor cell transcription factor SOX4 and extracellular matrix component tenascin C (29). A few miRNAs have been identified to target glycosylation enzymes to date. miR-378 targeted UDP- $N$-acetyl-D-galactosamine: polypeptide $\mathrm{N}$-acetylgalactosaminyltransferase 7 and enhanced the glycosylation of nephronectin, a ligand for integrin $\alpha 8 \beta 1$ (30). miR-148 targeted the enzyme core $1 \beta 1,3$-galactosyltransferase 1 and modulated $\operatorname{IgA} 1 O$-glycosylation and the level of secreted galactose-deficient IgA1 (31). miR-199a targeted the glycosylation enzyme ST6GAL1, reduced the sialylation of Necl-2, indirectly reduced the protein level of Necl-2, and enhanced ErbB2/ErbB3 signaling (32). It was reported that miR-493 inhibited the formation of metastatic foci and thus prevented liver metastasis of colon cancer cells (33). miR-493, was found to be reduced in lung cancer cells and tissues and to suppress cell invasion and proliferation by blocking E2F1 (34). miR-493 inhibited cell invasion and migration by blocking FZD4 and RhoC in bladder cancer (35).

miR-493-5p is prominently upregulated in male breast cancer (36). However, miR-493, as analyzed by NanoString's nCounter human miRNA expression profiling, was downregulated in TNBCs vs. normal breast tissues (37). The role of miR-493-5p remains unclear in breast cancer cell lines and in breast cancer at present. In the present study, we showed that the level of miR-493-5p, a potentially candidate tumorsuppressor miRNA, was low in MDA-MB-231 cells and breast cancer tissues. Introduction of miR-493-5p into MDA-MB-231 cells resulted in a reduction in tumor cell invasion and tumorigenicity. Furthermore, miR-493-5p was found to target the 3'-UTR of FUT4, causing decreased FUT4 expression at the mRNA and protein levels, thus controlling the invasiveness of breast cancer cells. This result indicated that miR-493-5p may serve as a specific modulator of human breast cancer invasion and tumorigenicity through targeting FUT4.

In summary, the FUT4 gene was demonstrated to regulate the invasiveness and tumorigenicity of human breast cancer. The aberrant expression of miR-493-5p was associated with breast cancer invasiveness and tumorigenicity. Moreover, we found that miR-493-5p targeted the 3'-UTR of FUT4 mRNA and reduced the protein expression level of FUT4. Our results provide new insight into the mechanisms underlying the invasion and tumorigenicity of breast cancer, and the application of miR-493-5p may be a potential strategy for breast cancer therapeutics.

\section{Acknowledgements}

The present study was supported by grants from the National Natural Science Foundation of China (81271910, 81472014), and from the Natural Science Foundation of Liaoning Province, China (2014023043).

\section{References}

1. Jemal A, Siegel R, Ward E, Murray T, Xu J and Thun MJ: Cancer statistics, 2007. CA Cancer J Clin 57: 43-66, 2007.
2. He D, Wang C, Cao L, Zhou J, Huang J and Fang H: Epidemic trend of female breast cancer incidence in Min Hang District, Shanghai. China Cancer 13: 108-110, 2010.

3. Stellrecht CM, Vangapandu HV, Le XF, Mao W and Shentu S: ATP directed agent, 8-chloro-adenosine, induces AMP activated protein kinase activity, leading to autophagic cell death in breast cancer cells. J Hematol Oncol 7: 23, 2014.

4. Apweiler R, Hermjakob $\mathrm{H}$ and Sharon $\mathrm{N}$ : On the frequency of protein glycosylation, as deduced from analysis of the SWISS-PROT database. Biochim Biophys Acta 1473: 4-8, 1999.

5. Spiro RG: Protein glycosylation: Nature, distribution, enzymatic formation, and disease implications of glycopeptide bonds. Glycobiology 12: 43R-56R, 2002.

6. Desiderio V, Papagerakis P, Tirino V, Zheng L, Matossian M, Prince ME, Paino F, Mele L, Papaccio F, Montella R, et al: Increased fucosylation has a pivotal role in invasive and metastatic properties of head and neck cancer stem cells. Oncotarget 6: 71-84, 2015.

7. Wang QY, Guo P, Duan LL, Shen ZH and Chen HL: $\alpha-1,3-$ Fucosyltransferase-VII stimulates the growth of hepatocarcinoma cells via the cyclin-dependent kinase inhibitor $\mathrm{p} 27^{\mathrm{Kipl}}$. Cell Mol Life Sci 62: 171-178, 2005.

8. Hiller KM, Mayben JP, Bendt KM, Manousos GA, Senger K, Cameron HS and Weston BW: Transfection of alpha $(1,3)$ fucosyltransferase antisense sequences impairs the proliferative and tumorigenic ability of human colon carcinoma cells. Mol Carcinog 27: 280-288, 2000.

9. Sanchez-Mejias A and Tay Y: Competing endogenous RNA networks: Tying the essential knots for cancer biology and therapeutics. J Hematol Oncol 8: 30, 2015.

10. Thomson DW, Bracken CP and Goodall GJ: Experimental strategies for microRNA target identification. Nucleic Acids Res 39: 6845-6853, 2011.

11. Calin GA and Croce CM: MicroRNA-cancer connection: The beginning of a new tale. Cancer Res 66: 7390-7394, 2006.

12. Croce CM: miRNAs in the spotlight: Understanding cancer gene dependency. Nat Med 17: 935-936, 2011.

13. Hurst DR, Edmonds MD, Scott GK, Benz CC, Vaidya KS and Welch DR: Breast cancer metastasis suppressor 1 up-regulates miR-146, which suppresses breast cancer metastasis. Cancer Res 69: 1279-1283, 2009.

14. Liu S, Goldstein RH, Scepansky EM and Rosenblatt M: Inhibition of rho-associated kinase signaling prevents breast cancer metastasis to human bone. Cancer Res 69: 8742-8751, 2009.

15. Phua YW, Nguyen A, Roden DL, Elsworth B, Deng N, Nikolic I, Yang J, Mcfarland A, Russell R, Kaplan W, et al: MicroRNA profiling of the pubertal mouse mammary gland identifies miR-184 as a candidate breast tumour suppressor gene. Breast Cancer Res 17: 83, 2015.

16. Lattová E, Tomanek B, Bartusik D and Perreault H: N-glycomic changes in human breast carcinoma MCF-7 and T-lymphoblastoid cells after treatment with herceptin and herceptin/Lipoplex. J Proteome Res 9: 1533-1540, 2010.

17. Shu H, Zhang S, Kang X, Li S, Qin X, Sun C, Lu H and Liu Y: Protein expression and fucosylated glycans of the serum haptoglobin- $\beta$ subunit in hepatitis B virus-based liver diseases. Acta Biochim Biophys Sin 43: 528-534, 2011.

18. Mejías-Luque R, López-Ferrer A, Garrido M, Fabra A and de Bolós C: Changes in the invasive and metastatic capacities of HT-29/M3 cells induced by the expression of fucosyltransferase 1. Cancer Sci 98: 1000-1005, 2007.

19. Vasseur JA, Goetz JA, Alley WR Jr and Novotny MV: Smoking and lung cancer-induced changes in N-glycosylation of blood serum proteins. Glycobiology 22: 1684-1708, 2012.

20. Julien S, Ivetic A, Grigoriadis A, QiZe D, Burford B, Sproviero D, Picco G, Gillett C, Papp SL, Schaffer L, et al: Selectin ligand sialyl-Lewis $\mathrm{x}$ antigen drives metastasis of hormone-dependent breast cancers. Cancer Res 71: 7683-7693, 2011.

21. Yan X, Lin Y, Liu S, Aziz F, Yan Q. Fucosyltransferase IV (FUT4) as an effective biomarker for the diagnosis of breast cancer. Biomed Pharmacother 70: 299-304, 2015.

22. Weston BW, Hiller KM, Mayben JP, Manousos GA, Bendt KM, Liu R and Cusack JC Jr: Expression of human alpha(1,3)fucosyltransferase antisense sequences inhibits selectin-mediated adhesion and liver metastasis of colon carcinoma cells. Cancer Res 59: 2127-2135, 1999.

23. Palumberi D, Aldi S, Ermini L, Ziche M, Finetti F, Donnini S and Rosati F: RNA-mediated gene silencing of FUT1 and FUT2 influences expression and activities of bovine and human fucosylated nucleolin and inhibits cell adhesion and proliferation. J Cell Biochem 111: 229-238, 2010. 
24. Guo Q, Guo B, Wang Y, Wu J, Jiang W, Zhao S, Qiao S and $\mathrm{Wu}$ Y: Functional analysis of $\alpha 1,3 / 4$-fucosyltransferase VI in human hepatocellular carcinoma cells. Biochem Biophys Res Commun 417: 311-317, 2012.

25. Lim LP, Lau NC, Garrett-Engele P, Grimson A, Schelter JM, Castle J, Bartel DP, Linsley PS and Johnson JM: Microarray analysis shows that some microRNAs downregulate large numbers of target mRNAs. Nature 433: 769-773, 2005.

26. Naidu S, Magee P and Garofalo M: MiRNA-based therapeutic intervention of cancer. J Hematol Oncol 8: 68, 2015.

27. Voorhoeve PM, le Sage C, Schrier M, Gillis AJ, Stoop H, Nagel R, Liu YP, van Duijse J, Drost J, Griekspoor A, et al: A genetic screen implicates miRNA-372 and miRNA-373 as oncogenes in testicular germ cell tumors. Cell 124: 1169-1181, 2006.

28. Ma L, Teruya-Feldstein J and Weinberg RA: Tumour invasion and metastasis initiated by microRNA-10b in breast cancer. Nature 449: 682-688, 2007.

29. Tavazoie SF, Alarcón C, Oskarsson T, Padua D, Wang Q, Bos PD, Gerald WL and Massagué J: Endogenous human microRNAs that suppress breast cancer metastasis. Nature 451: 147-152, 2008

30. Kahai S, Lee SC, Lee DY, Yang J, Li M, Wang CH, Jiang Z, Zhang Y, Peng C and Yang BB: MicroRNA miR-378 regulates nephronectin expression modulating osteoblast differentiation by targeting GalNT-7. PLoS One 4: e7535, 2009.

31. Serino G, Sallustio F, Cox SN, Pesce F and Schena FP: Abnormal miR-148b expression promotes aberrant glycosylation of IgA1 in IgA nephropathy. J Am Soc Nephrol 23: 814-824, 2012.
32. Minami A, Shimono Y, Mizutani K, Nobutani K, Momose K, Azuma T and Takai Y: Reduction of the ST6 $\beta$-galactosamide $\alpha$-2,6-sialyltransferase 1 (ST6GAL1)-catalyzed sialylation of nectin-like molecule 2/cell adhesion molecule 1 and enhancement of ErbB2/ErbB3 signaling by microRNA-199a. J Biol Chem 288: 11845-11853, 2013.

33. Okamoto K, Ishiguro T, Midorikawa Y, Ohata H, Izumiya M, Tsuchiya N, Sato A, Sakai H and Nakagama H: miR-493 induction during carcinogenesis blocks metastatic settlement of colon cancer cells in liver. EMBO J 31: 1752-1763, 2012.

34. Gu Y, Cheng Y, Song Y, Zhang Z, Deng M, Wang C, Zheng G and He Z: MicroRNA-493 suppresses tumor growth, invasion and metastasis of lung cancer by regulating E2F1. PLoS One 9: e102602, 2014

35. Ueno K, Hirata H, Majid S, Yamamura S, Shahryari V, Tabatabai ZL, Hinoda Y and Dahiya R: Tumor suppressor microRNA-493 decreases cell motility and migration ability in human bladder cancer cells by downregulating RhoC and FZD4. Mol Cancer Ther 11: 244-253, 2012.

36. Lehmann U, Streichert T, Otto B, Albat C, Hasemeier B, Christgen H, Schipper E, Hille U, Kreipe HH and Länger F: Identification of differentially expressed microRNAs in human male breast cancer. BMC Cancer 10: 109, 2010.

37. Cascione L, Gasparini P, Lovat F, Carasi S, Pulvirenti A, Ferro A, Alder H, He G, Vecchione A, Croce CM, et al: Integrated microRNA and mRNA signatures associated with survival in triple negative breast cancer. PLoS One 8: e55910, 2013. 